\title{
Research of the Psychological Interconnection between the Basic Odors and the Emotional State of a Human Being
}

\author{
Tatiana Nikolaevna Berezina ${ }^{1,2}$ \\ ${ }^{1}$ Sholokhov Moscow State University for the Humanities/Department of General and Applied Psychology. \\ Moscow, Russia \\ ${ }^{2}$ Moscow State University of Psychology \& Education/Department of extreme psychology. Moscow, Russia \\ Correspondence: Tatiana Nikolaevna Berezina, st.Young Leninists, 105/1 - 2, 109439, Moscow, Russia. E-mail: \\ russian-science_info@mail.ru
}

Received: August 18, 2014 Accepted: September 23, 2014 Online Published: October 30, 2014

doi:10.5539/ass.v10n21p159

URL: http://dx.doi.org/10.5539/ass.v10n21p159

\begin{abstract}
Psychological interrelation between the base odors (according to Amoore) and the base emotional states of human beings (according to Ekman) is established. The subjects were offered samples of the basic odors. Their task was to associate these odors with one of the proposed basic emotions. The analysis of variance showed the significant association of putrid odor with the emotion of disgust, the odor of mint with the emotion of surprise, and the odor of musk with the emotion of anger. Other odors can cause two or three emotions. Based on the theoretical analysis, the article proposes to associate flowery odor with the emotion of joy, the odor of fruit with the emotion of pleasure, the pungent odor with the emotion of fear, and the odor of camphor with the emotion of sadness. Hypothesis of the existence of olfactory-emotional language allowing to create the emotional tone of the odor of any object, is offered.
\end{abstract}

Keywords: odors, base odors, emotions, base emotions

\section{Introduction}

The interrelation of odors and the emotional sphere is well known. This relationship is caused by both physiological and psychological mechanisms. Researchers emphasize the direct relationship between olfaction and the limbic system, which is responsible for the generation of human emotions. One of consequences of that relationship is considered the emotional component of odors. Some researchers suggest that the direct relationship with the limbic system explains the expressed emotional component of olfactory sensations. Odors can cause pleasure or disgust (hedonistic components), influencing the affective condition of the organism. D. Chen and P. Dalton (Chen \& Dalton, 2005) have written on the existence of a relationship between emotions and olfactory perception. In empirical studies, D. Chen and J.Haviland-Jones have shown that it is possible to define human emotional states according to odor of the person. In particular, it is possible to distinguish the state of fear from the state of pleasure (Chen \& Haviland-Jones, 2000). Using odors, it is also possible to change the emotional state of a person. To trigger a change of mood, researchers have used either the odors of other people who are experiencing a strong emotion or erotic odors, e.g., the odor of sex steroids (Bensafi et al., 2004). It has also been shown that the ability to distinguish between the odors (i.e., the differentiation of pleasant odors from unpleasant) depends on the presence or absence of emotional disorders as the ability to differentiate worsens during depression and is restored upon treatment (Pause et al., 2003). According to R. Herz, odors relate to human memory: an odor can trigger flashbacks and thus cause the same emotional state that was present at the original moment of perception (Herz, 2005).

The relationship between odors and emotions is well known, but unstudied remains the relationship between the base odors and emotions. The concept of the base odors assumes that all aromas surrounding us are composed of several primary (base) odors. There are several classifications of the base odors. In one of the very first classifications - so-called Henning's prism - the six base odors are defined: flowery, fruity (ethereal), decomposed, burned, spicy and rubber odors (Henning, 1924). The most advanced contemporary theory of olfaction - the stereochemical theory - is based on the seven base odors, three of which correspond to the Henning's classification: flowery, ethereal, and decomposed (Amoore, 1965). The authors of this theory have made significant contributions 
in the analysis of aromatic compounds and mechanisms of their interaction with receptors of the olfactory analyzer. They have demonstrated the existence of seven olfactory receptor sites distinguished in the structural formulas of the substances capable of interacting with these receptor sites. According to the stereochemical theory of olfaction, the base odors differ from one another, not chemically, but by the presence of odorate molecules of definitely aligned structures in their composition. Therefore, substances from various chemical groups having the necessary stereostructure, can cause sensations of the same base odor. The base odors are named according to the most typical substances causing them: Flowery (rose, lavender), Ethereal (ether), Musky (musk), Camphoric (camphor, eucalyptus), Minty (mint, menthol), Caustic (acetic acid), and Decomposed (rotten eggs).

Among the base odors, several have repeatedly been the object of scientific research, including researches on the ability of odors to influence the human emotions and behavior. For example, it has been shown that a flowery odor (lavender) can improve night dream of subjects and lead to improvement of mood at the moment of awakening (Goel, 2004). A similar effect has been found for pleasant natural odors: they improved subjects' mood and reduced their vigilance level during the conducted experiment (Weber \& Heuberger, 2008). It has been shown that peppermint can influence not only emotions but also help solving clerical problems: under the influence of peppermint, subjects have shown improved accuracy and increased speed in text printing. The quantity of errors decreased, and the subjects ordered words alphabetically faster under the influence of minty odor. The authors attributed this effect to the attention improvement (Barker et al., 2003).

The influence of the two base odors on emotions arising during dreaming, has been demonstrated as well: a pleasant odor (e.g., the smell of roses) improved the mood of the subject and positively changed the nature of the dream. An unpleasant odor (e.g., the smell of rotten eggs), instead, promoted the occurrence of negative emotions and unpleasant experiences in the dream (Schredl et al., 2009).

However, the question regarding the relationship between the base odors and the base emotions remains. The analysis of the base emotions was started in 1860, when C. Darvin attempted to distinguish the "true and original" forms of human emotional behavior. Continuing these studies, Ekman differentiated the six base emotions based on human expressions equally perceived by the representatives of various cultures: anger, disgust, fear, joy, grief, and surprise (Ekman, 1972). However, there are many classifications of the base emotions (the review of classifications is presented in Ortony and Turner, 1990). We will simply note that many authors distinguish the two positive base emotions: pleasure and joy.

Earlier we have shown that the representation of mental images affect the state of the human organism (Berezina, 2009). In our previous study, we also studied associative relations between emotions and odors. The subjects were asked to state the base odors they associated with each of the base emotions. We used the list of the base emotions by Ekman. Although, we distinguish joy and pleasure as two different emotions. We have shown that the human imagination steadily bounds some base emotions to the base odors. In this case, we were interested in flowery, fruity, decomposed, and mint odors (Berezina, 2012). However, our previous study examined the relationship between words for odors and words for emotions instead of the relationship between emotions and actual odors, which has not been studied yet.

The present study is devoted to understanding the relationship between the real base odors and the base emotions. We based our definition of the base odors on J. Amoore's classification, but instead of ethereal odor (the source of which is diethyl ether), we used fruity odor, in accordance with Henning, who considered the action of fruity odor to be similar to that of ethereal odor.

\section{Research Methodology}

Subjects. Subjects consisted of 100 young adults (50 male), from 18 to 35, with no deviations in physical or mental development. All were students of humanitarian specialties.

Methods:

1. Methods of chemical action. Leaflets of a filter paper with the putted sample of the base odor were used as a stimuli material. Stimuli materials were placed in the closed packages

File 1 - A source of the base flowery odor - aromatic oil "rose" - 1 drop.

File 2 - A source of the base fruity (etheric) odor - aromatic oil "muscatel" (grape) - 1 drop.

File 3 - A source of the base mint odor - aromatic oil "mint" - 1 drop.

File 4 - A source of the base pungent odor - acetic acid - 1 drop.

File 5 -A source of the base camphor odor - camphor -1 drop. 
File 6 - A source of the base musk odor - animal unpurified musk - castor extract - 1 drop.

File 7 - A source of the base decomposed odor - rotten egg - 1 drop.

2. The list of the base emotions. There were 7 paper leaflets, on each of which one base emotion was written: Joy, Pleasure, Surprise, Fear, Grief, Anger, and Disgust.

Procedure. Odors (flowery, fruity, mint, and decomposed) were placed on the leaf of a filter paper and packed into the file on the morning of the day of the experiment. Pungent, camphoric and musky odors were placed on the paper immediate prior to the experiment.

The stimuli material, in the form of papers packed into the file with odors, was displayed on a long table. Each stimulus was approximately $50 \mathrm{~cm}$ from the next.

Each subject received instructions, in which he or she was informed of the existence of the base odors (all the odors were listed) and the base emotions (all the emotions were listed). The subject also received the paper leaflets with the written emotions.

The subject passed along the table, smelling the base odors.

At repeated passage, the subject matched the paper with the emotion that the smell caused, near a source.

If necessary, the subject passed down the table once again, smelled the odors and changed leaflets with emotions until satisfied with the result.

Half of all subjects began movement from file 1 (smell of rose); the other half began movement from file 7 (smell of rotten eggs). This procedure was performed to compensate for the effect of the first sensory impression.

Data analyses. We formed 7 "variable objects" matrices, according to the quantity of researched stimulus. In each matrix, the odor acted as the independent variable, and emotions acted as dependent variables.

In the first matrix, the flowery odor acted as an independent variable.

Categorical factor: Flowery odor. 1 level - the trial subject did not smell a rose, i.e., he smelled all other odors, 2 level - the trial subject smelled a rose.

Dependent variables - emotions.

Dependent variable 1 - the emotion of joy. If the subject considered this odor to be associated with the emotion of joy, 1 point was given. If this odor was associated with any other emotion, 0 points were given.

Dependent variable 2 - the emotion of pleasure; it was estimated similarly.

Dependent variable 3 - the emotion of surprise; it was estimated similarly.

Dependent variable 4 - emotion of fear; it was estimated similarly.

Dependent variable 5 - the emotion of grief; it was estimated similarly.

Dependent variable 6 - the emotion of anger; it was estimated similarly.

Dependent variable 7 - the emotion of disgust; it was estimated similarly.

The subsequent matrices (second - seventh) were formed similarly: in each matrix, the base odor acted as a categorical factor.

The one-way ANOVA test from a package of standard statistical programs Statistica 6 was used for an estimation of the influence reliability.

\section{Results}

The base odors cause the base emotions in the subjects. The influence of odors on the emotional sphere is highly subjective, and any base odor can cause almost any emotion in the subject, depending on the conditions, which have not been examined in the present study. However, each odor caused similar emotions in the majority of subjects. The results of odor correlation with the emotions they caused are presented as percentages in Table 1 .

It is necessary to consider that in causal distributions of emotions in relationship to odors, each emotion would cause in $14-15 \%$ of cases. If emotion is caused more often, it is possible to assume that this odor causes this emotion in the subject. If emotion is met less often, it is possible to assume that this odor suppresses this human emotion.

The table 1 shows that each odor caused a spectrum of emotional reactions. The flowery odor mainly caused positive emotions (joy and pleasure); it also caused one negative emotion (grief) and suppressed other negative emotions (anger, fear, and disgust). 
Table 1. The correlation between the base odors and the emotions - total sampling (\%)

\begin{tabular}{|c|c|c|c|c|c|c|c|}
\hline & \multicolumn{7}{|c|}{ emotions } \\
\hline & joy & pleasure & surprise & anger & grief & fear & disgust \\
\hline \multirow{8}{*}{$\begin{array}{l}\text { odor } \\
\mathrm{S}\end{array}$} & Flowery- & Flowery & Flowery & Flowery & Flowery & Flowery- & Flowery- \\
\hline & $30 \%$ & $-32 \%$ & $-13 \%$ & $-6 \%$ & $16 \%$ & $2 \%$ & $1 \%$ \\
\hline & Fruity- 39\% & Fruity- $42 \%$ & Fruity- $12 \%$ & Fruity- 2\% & Fruity- $4 \%$ & Fruity- $0 \%$ & Fruity- $1 \%$ \\
\hline & Mint- 19\% & Mint- $15 \%$ & Mint -fruity & Mint- $7 \%$ & Mint- 17\% & Mint- 7\% & Mint- $0 \%$ \\
\hline & Musk- $2 \%$ & Musk- $1 \%$ & $35 \%$ & Musk- $31 \%$ & Musk- 18\% & Musk- $22 \%$ & Musk- $14 \%$ \\
\hline & $\begin{array}{l}\text { Camphor- } \\
7 \%\end{array}$ & $\begin{array}{l}\text { Camphor- } \\
7 \%\end{array}$ & $\begin{array}{l}\text { Musk- } 12 \% \\
\text { Camphor- }\end{array}$ & $\begin{array}{l}\text { Camphor- } \\
14 \%\end{array}$ & $\begin{array}{l}\text { Camphor- } \\
25 \%\end{array}$ & $\begin{array}{l}\text { Camphor- } \\
29 \%\end{array}$ & $\begin{array}{l}\text { Camphor- } \\
5 \%\end{array}$ \\
\hline & $\begin{array}{l}\text { Pungent- } \\
1 \%\end{array}$ & $\begin{array}{l}\text { Pungent- } \\
2 \%\end{array}$ & $\begin{array}{l}13 \% \\
\text { Pungent- }\end{array}$ & $\begin{array}{l}\text { Pungent- } \\
28 \%\end{array}$ & $\begin{array}{l}\text { Pungent- } \\
16 \%\end{array}$ & $\begin{array}{l}\text { Pungent- } \\
32 \%\end{array}$ & $\begin{array}{l}\text { Pungent- } \\
8 \%\end{array}$ \\
\hline & $\begin{array}{l}\text { Decompose } \\
\text { d- } 2 \%\end{array}$ & $\begin{array}{l}\text { Decompose } \\
\text { d- } 1 \%\end{array}$ & $\begin{array}{l}13 \% \\
\text { Decompose } \\
\text { d- } 2 \%\end{array}$ & $\begin{array}{l}\text { Decompose } \\
\text { d- } 12 \%\end{array}$ & $\begin{array}{l}\text { Decompose } \\
\text { d- } 4 \%\end{array}$ & $\begin{array}{l}\text { Decompose } \\
\text { d- } 8 \%\end{array}$ & $\begin{array}{l}\text { Decompose } \\
\text { d- } 71 \%\end{array}$ \\
\hline
\end{tabular}

The fruity odor also caused positive emotions (joy and pleasure) and suppressed all negative emotions.

The mint odor caused the emotions of surprise; the emotions of joy and grief were more rare; it suppressed negative emotions (anger, fear and disgust).

The musk odor (animal unpurified musk) caused only negative emotions and suppressed joy and pleasure.

The camphor odor caused negative emotions of grief and fear, although it did not cause negative emotions of disgust; it suppressed emotions of joy and pleasure.

The pungent odor caused emotions of fear and anger; it did not cause the emotions of disgust but suppressed the positive emotions of joy and pleasure.

The decomposed odor caused the emotions of disgust and suppressed almost all other emotions.

We estimated the reliability of causal reactions according to one-way ANOVA. The results of the factorial analysis are presented in Table 2.2- 2.8

Table 2. The results of one-way ANOVA test for the flowery odor

\begin{tabular}{ccccccccc}
\hline odors & statistics & joy & pleasure & surprise & anger & grief & fear & disgust \\
\hline \multirow{2}{*}{ flowery } & $\mathrm{F}$ & 24,28 & 31,14 & 0,157 & 6,411 & 0,279 & 14,64 & 17,41 \\
& $\mathrm{p}$ & $0,000^{*}$ & $0,000^{*}$ &, 692 & 0,012 & 0,597 & $0,000^{*}$ & $0,000^{*}$ \\
\hline
\end{tabular}

Table 3. The results of one-way ANOVA test for the fruity odor

\begin{tabular}{ccccccccc}
\hline odors & statistics & joy & pleasure & surprise & anger & grief & fear & disgust \\
\hline \multirow{2}{*}{ fruity } & $\mathrm{F}$ & 63,29 & 81,49 &, 497 & 14,42 & 10,20 & 19,94 & 17,41 \\
& $\mathrm{p}$ & $0,000^{*}$ & $0,00^{*}$ & 0,481 & $0,000^{*}$ & $0,001^{*}$ & $0,000^{*}$ & $0,000^{*}$ \\
\hline
\end{tabular}

Table 4. The results of one-way ANOVA test for the mint odor

\begin{tabular}{ccccccccc}
\hline odors & statistics & joy & pleasure & surprise & anger & grief & fear & disgust \\
\hline \multirow{2}{*}{$\operatorname{mint}$} & $\mathrm{F}$ & 2,118 & 0,048 & 43,29 & 4,923 & 0,701 & 5,080 & 20,18 \\
& $\mathrm{p}$ & 0,146 & 0,826 & $0,000^{*}$ & 0,027 & 0,403 & 0,025 & $0,000^{*}$ \\
\hline
\end{tabular}


Table 5. The results of one-way ANOVA test for the musk odor

\begin{tabular}{ccccccccc}
\hline odors & statistics & joy & pleasure & surprise & anger & grief & fear & disgust \\
\hline \multirow{2}{*}{ musk } & $\mathrm{F}$ & 14,64 & 17,18 &, 497 & 28,33 & 1,313 & 5,700 &, 017 \\
& $\mathrm{p}$ &, $000^{*}$ &, $000^{*}$ &, 481 &, $000^{*}$ &, 252 &, 017 &, 895 \\
\hline
\end{tabular}

Table 6. The results of one-way ANOVA test for the camphor odor.

\begin{tabular}{lllllllll}
\hline camphor & $\mathrm{F}$ & 5,080 & 5,080 & 0,157 & 0,002 & 11,08 & 21,19 & 8,478 \\
& $\mathrm{p}$ & 0,025 & 0,025 & 0,692 & 0,965 & $0,001^{*}$ & $0,000^{*}$ & $0,004^{*}$ \\
camphor & $\mathrm{F}$ & 5,080 & 5,080 & 0,157 & 0,002 & 11,08 & 21,19 & 8,478 \\
\hline
\end{tabular}

Table 7. The results of one-way ANOVA test for the pungent odor

\begin{tabular}{ccccccccc}
\hline odors & statistics & joy & pleasure & surprise & anger & grief & fear & disgust \\
\hline \multirow{2}{*}{ pungent } & $\mathrm{F}$ & 17,18 & 14,64 & 0,157 & 16,20 & 0,279 & 31,14 & 2,788 \\
& $\mathrm{p}$ & $0,000^{*}$ & $0,000^{*}$ & 0,692 & $0,000^{*}$ & 0,597 & $0,000^{*}$ & 0,095 \\
\hline
\end{tabular}

Table 8. The results of one-way ANOVA test for the decomposed odor

\begin{tabular}{ccccccccc}
\hline odors & statistics & joy & pleasure & surprise & anger & grief & fear & disgust \\
\hline \multirow{2}{*}{ decomposed } & $\mathrm{F}$ & 14,64 & 17,18 & 14,64 & 0,440 & 10,20 & 3,774 & 530,9 \\
& $\mathrm{p}$ & $0,000^{*}$ & $0,000^{*}$ & $0,000^{*}$ & 0,507 & $0,001^{*}$ & 0,052 & $0,00^{*}$ \\
\hline
\end{tabular}

Independent variable is odor (column), dependent variable is emotion (line).

$\mathrm{p}$ - level of significance

$* \leq 0,01$

The tables show that the variance analysis confirms the previous results. Thus, the flowery odor authentically causes the emotions of joy and pleasure and suppresses emotions of fear and disgust. The strongest reaction is the emotion of pleasure.

The fruity odor authentically causes emotions of joy and pleasure and suppresses emotions of fear, anger, grief and disgust. The strongest reaction is the emotion of pleasure.

The mint odor authentically causes the emotion of surprise and suppresses disgust. The strongest reaction is surprise.

The musk odor authentically causes the emotion of anger and suppresses joy and pleasure. The strongest reaction is anger.

The camphor odor authentically causes the emotions of fear, grief, and disgust. The strongest emotion is fear.

The pungent odor authentically causes the emotions of fear and anger and suppresses emotions of joy and pleasure. The strongest reaction is fear.

The decomposed odor authentically causes the emotion of disgust and suppresses the emotions of joy, pleasure, surprise, and grief.

\section{Discussion}

The interrelation of pleasant odors with positive emotions and of unpleasant odors with the negative emotions is obvious, following from ordinary life experience and confirmed by the work of contemporary researchers (Weber \& Heuberger, 2008; Schredl et al., 2009).

However, we assume that this relationship is deeper and stronger than it first appears, and it is possible that there is an unequivocal relationship between the base odors and the emotions they cause. 
In our study, this interrelationship is traced, and variance analysis has shown the binding of distinguishing the base odors to the base emotions more precisely. Relying on our data, we offer the following connections. In part, the existence of these relationships is confirmed by other authors, and some connections are unequivocal. The mint odor authentically causes only one emotion (i.e., surprise) and coincides with the effect of peppermint described in the literature: the human attention improves under the influence of this odor, and subjects are more successful in solving verbal problems (Barker et al., 2003). A similar effect accompanies the occurrence of the emotion of surprise and interest in the subject.

The pungent odor authentically causes only the emotion of disgust, which is also confirmed in the literature. Researchers have shown that the smell of rotten eggs during a dream promotes the occurrence of negative emotions and unpleasant experiences (Schredl et al., 2009).

The musk odor authentically causes only the emotion of anger.

In other cases, the base odor authentically causes two or three similar emotions. There are several explanations for this. For example, the flowery and fruity odors authentically cause both the emotions of joy and pleasure because it is difficult for the subject to distinguish between these two emotions. For many people, these appear to be one and the same emotion. We will repeat that Ekman does not distinguish between these two emotions. For others, these emotions are distinct, but they cannot explain in what manner. The complexity of the subjective separation of emotions has led to an overshoot of associative fields of flowery and fruity odors. Moreover, we took a sweetish smell of grapes (muscatel), as stimuli of a fruity odor. Most likely, when using other fruity odors, differences in associations would act more distinctly.

We have assumed that the flowery odor is associated with the positive emotion of joy and that the fruity odor is associated with the positive emotion of pleasure. The relationship between the emotion of pleasure and a fruity odor can be explained by that fact that fruits are one of the principal types of food for many vertebrata, including our immediate human ancestors, anthropoids. Perhaps it is due to this fact that a fruity odor causes positive emotions. It is also important to note that in the stereochemical theory, the fruity odor acts similarly to the ethereal odor. Amoore, in general, preferred to name this base odor ethereal and to consider the diethyl ether as a sample of the odor. The diethyl ether belongs to substances causing human anesthesia reaction. The same substances in the human body promote anesthesia and cause pleasure. Accordingly, we have correlated the fruity odor with the emotion of pleasure and the flowery odor with the emotion of joy. According to other researchers, flowery odors cause positive emotions: while sleeping (Schredl et al., 2009), flowery odors improve a night dream and mood at the moment of awakening (Goel, 2004).

The pungent odor also authentically causes two emotions: fear and anger. This occurs because the emotions of anger and fear are bound together both physiologically and psychologically. The same situation can cause both fear and anger. The nervous centers are found in the hypothalamus, the stimulation of which may cause fear or anger, depending on the intensity. Therefore, subjectively at the odor perception, associative fields of anger and fear emotions can be blocked. Nevertheless, within the limits of the odor-emotional language model, we will connect a pungent odor to the emotion of fear, while the emotion of anger has been connected earlier to the musky odor. In other our works, we studied the influence of pungent odors on the emotional behavior of animals: dogs (Berezina, 2009 a) and insects (Berezina, 2013). We showed that in both dogs and ants, the odor of acetic acid authentically causes the behavioral fear reaction, while the occurrence of anger reaction (dogs) did not reach reliability level.

The most difficult to interpret are the emotional reactions to camphor odor, as it causes three negative emotions (fear, grief, and disgust). As we have already connected fear and disgust to other odors, it is necessary to connect the camphor odor to the emotion of grief. In our other work in which subjects matched olfactory associations to the base odors, the emotion of grief was most often connected with odors of bitterness and burnt odors (Berezina, 2012); according to Henning, the burned odor belongs to the base odors.

In our work, we used the most recognized typologies: the classification of the base odor according to Amoore and the classification of the base emotions according to Ekman. However, as we noted above, there are alternative classifications for both. Most likely, not all odors allocated by Amoore are the base odors. First, however, this does not refute the fact that there is a relationship between an original base odor and a base emotion; second, an appreciable part of odors, according to Amoore classification, most likely belongs to the base odors. It may be disputable whether odors cause several base emotions.

Furthermore, when speaking about the relationship of the base odor and emotions, we assume that this relationship is, to a great degree, unconscious, i.e., often odors are not perceived as aromas, and emotions do not reach the level of obvious experiences. 
It is well known that the research of the base emotions is associated with the demand of "an emotional priority thesis". According to Griffiths, emotional priority means a recognition of independence of emotional responses from the rational estimations of things; thus, we dare to be afraid of things whose safety we do not doubt and to be irritated on perfect not guilty things, i.e., the emotional answer of an organism is a part of the stimulus and does not depend on a rational estimation of the available situation (Griffiths, 2003). In other words, some stimuli always cause a particular emotion, regardless of whether it is rational at the present moment. In Ekman's work, similar ideas were embodied in the concept of the "mechanism of an automatic estimation," in which the cognitive subsystem serves to define the capacity of the stimulus to cause a base emotion and to operate independently from the cognitive system generating conscious, verbally expressed estimations of the same stimulus (Ekman, 1980).

According to "bi - or multimodal models of emotions," it is assumed that processing of the emotional information from the stimulus is performed at two or more levels. Thus, at the low level, the formation of the emotional response is performed without conscious participation (e.g., LeDoux, 1996).

The existence of such unconscious emotions, being the part of visual perception, is convincingly shown by the Netherlands psychologists from the Tilburg Institute for Behavioral Economics Research (Ruys and Stapel, 2008). In a series of original experiments, subjects were rapidly shown pictures causing disgust, fear, or neutral emotions at a subconscious level (so that they could not realize the maintenance) and noted the corresponding change of mood.

In other studies, images of snakes, spiders, colors, mushrooms, etc., were shown to subjects so that the person could not realize them; however, people with preliminarily revealed ophidiophobia showed a rising of skin conductivity in response to images of snakes, and people with a fear of spiders reacted similarly to images of spiders (Öhman \& Soares, 1994).

However, while the majority of such works are concerned with the perception of visual stimuli, in our research, the goal was to bind the occurrence of the base emotions with the action of the base odors. Nevertheless, it is possible to assume that nature has hardly created two different mechanisms for forming emotional responses to visual and olfactory stimuli.

In our early work, we assumed the evolutionary character of the relationship between odors and emotions (Berezina, 2012). We assumed the existence of an olfactory-emotional language, in which each base odor causes a base emotion. Thus, the perception of any subject will first include the olfactory perception, as sets of the base odors, and second, the subsequent complex emotional reaction constructed from a series of base odors. Thus, it is possible to explain the existence of the emotional tone of olfactory sensations. By definition, the emotional tone of olfactory sensations is a genetically primary and elementary form of emotion that accompanies any sensation. They are realized as emotional coloring, an original qualitative accent accompanying temperature, gustatory, sound, olfactory and other sensations. W. Wundt noted the existence of an emotional background of sensations, asserting that "emotional tone" generally represents a short designation of those simple feelings that we more or less regularly meet in connection with certain simple sensations (Wundt, 1874).

We hypothesize that the emotional tone of olfactory sensations can accompany more than elementary sensations, comparable with the base odors. However, because of the existence of odor-emotional language, any subject, and even any person or any social situation having this odor, will have the same emotional code.

It is possible to propose such a model. When first encountering an object, its smell, in the nasal cavity of the perceiving person, will break up into its base components. Thus, the unique olfactory characteristic of this object is formed.

Furthermore, the emotional answer to this stimulus, in the form of the set of the base emotions, is formed in the limbic system, which represents the original emotional tone of the base emotional sensations.

Thus, the subject will usually have this smell, and accordingly, there will be an emotional reaction. As the subject's smell is constant, the emotional tone from the smell of this subject will also be nearly identical.

\section{Summary and Conclusions}

The model of existence of olfactory-emotional language was offered to support the existence of an unequivocal connection between the base odors and the base emotions. The existence of such a connection explains the phenomenon of the emotional tone of sensations, which states that certain thermal, sound, olfactory, etc. sensations cause specific emotions. Our model assumes the existence of an emotional background in each base odor; thus, the base emotions will also be caused by the emotional tone. Such an organization of mental life assumes the existence of an emotional code for any object and not simply of the elementary odors. The person 
will perceive the aroma of any object as a set of the base odors. Each odor will cause the corresponding base emotion, which will create the unique emotional coloring of the object's perception. We assume that this process usually is not consciously realized by the person. The person may neither realize the actions of smells nor the arising emotional response. However, in specially organized conditions, it is possible to uncover the emotional effect of each base odor. Similar conditions have been reproduced in our empirical research. The subjects were given the seven base odors as stimuli and matched each base emotion to each odor. Relying on the data of the variance analysis and theoretical reasoning, we propose the following interrelationships: the flowery odor is connected to the emotion of joy, the fruity odor is connected to the emotion of pleasure, the mint odor is connected to the emotion of surprise, the musk odor is connected to the emotion of anger, the decomposed odor is connected to the emotion of disgust, the pungent odor is connected to the emotion of fear, and the camphor odor is connected to the emotion of grief.

\section{Reference}

Amoore, J. E. (1965). Current status of the theory of odor. Annals of the New York Academy of Sciences, 116, 457-476. http://dx.doi.org/10.1111/j.1749-6632.1964.tb45075.x

Barker, S., Grayhem, P., Koon, J., Perkins, J., Whalen, A., \& Raudenbush, B. (2003). Improved performance on clerical tasks associated with administration of peppermint odor. Perceptual and Motor Skills, 97, 1007-10. http://dx.doi.org/10.2466/pms.2003.97.3.1007

Bensafi, M., Brown, W. M., Kahn, R., \& Levenson, S. (2004). Sniffing human sex-steriod derived compounds modulates mood and memory and autonomic nervous system function in specific behavioral contexts. Behavioural Brain Research, 152, 11-22;

Berezina, T. N. (2009). The odor and image of alcohol as factors affecting functional state of a person. Voprosy Psikhologii, (4), 80-90.

Berezina, T. N. (2009a). The question of «fear odor» existence. Psychology, 1, c. 102-111.

Berezina, T. N. (2012). Interrelation of basic smells and basic emotions. Voprosy Psikhologii, (4), 106-116.

Berezina, T. N. (2013). Influence of smells on insects (on the example of a fear smell). NB: Psychology and psychotechnics, 8, 1-20. Retrieved from http://e-notabene.ru/psp/article_10610.html

Chen, D., \& Dalton, P. (2005). The Effect of Emotion and Personality on Olfactory Perception. Chemical Senses, 30, 345-35. http://dx.doi.org/10.1093/chemse/bji029

Chen, D., \& Haviland-Jones J. (2000). Human Olfactory communications of emotion. Perceptual Motor Skills, 91, 771-781. http://dx.doi.org/10.2466/pms.2000.91.3.771

Ekman, P. (1972). Emotions in the Human Face. New York: Pergamon Press.

Ekman, P. (1980). Biological \& cultural contributions to body \& facial movement in the expression of emotions. In A. O. Rorty (Ed.), Explaining Emotions. Berkeley: University of California Press.

Goel, N. (2004). Effects of Olfactory stimuli on subsequent human sleep. Wesleyan University.

Griffiths, P. E. (2003). Basic Emotions, Complex Emotions, Machiavellian Emotions. In A. Hatzimoysis (Ed.), Philosophy and the Emotions (pp. 39-67). Cambridge, CUP. http://dx.doi.org/10.1017/CBO978051155 0270.004

Henning, H. (1924). Der Geruch. Lpz.: Barth.

Herz, R. S. (2005). Odor-associative learning and emotion: effects on perception and behavior. Chemical Senses, 30(Suppl 1), 1250-51. http://dx.doi.org/10.1093/chemse/bjh209

LeDoux, J. (1996). The Emotional Brain: The Mysterious Underpinnings of Emotional Life. New York: Simon and Schuster.

Öhman, A., \& Soares, J. J. F. (1994). Unconscious anxiety: phobic responses to masked stimuli. Journal of Abnormal Psychology, 102, 121-132. http://dx.doi.org/10.1037/0021-843X.102.1.121

Ortony, A., \& Turner, T. J. (1990). What's basic about basic emotions? Psychological Review, 97, 315-331. http://dx.doi.org/10.1037/0033-295X.97.3.315

Pause, B. M, Raack, N., Sojka, B., Goder, R., Aldenhoff, J. B., \& Ferstl, R. (2003). Convergent and divergent effects of odors and emotions in depression. Psychophysiology, 40, 209-25. http://dx.doi.org/10.1111/14698986.00023 
Ruys, K., \& Stapel, D. (2008). The Secret Life of Emotions. Psychological Science, 19(4). http://dx.doi.org/10.1111/j.1467-9280.2008.02097.x

Schredl, M., Atanasova1, D., Hoërmann, K., Maurer, J. T., Hummel, T., \& Stuck, B. A. (2009). Information processing during sleep: the effect of olfactory stimuli on dream content and dream emotions. J. Sleep Res., 18, 285-290. http://dx.doi.org/10.1111/j.1365-2869.2009.00737.x

Weber, S., \& Heuberger, E. (2008). The Impact of Natural Odors on Affective States in Humans. Chemical Senses, 33(5), 441-447. http://dx.doi.org/10.1093/chemse/bjn011

Wundt, W. (1874/1945). Principles of Physiological Psychology. New York.

\section{Copyrights}

Copyright for this article is retained by the author(s), with first publication rights granted to the journal.

This is an open-access article distributed under the terms and conditions of the Creative Commons Attribution license (http://creativecommons.org/licenses/by/3.0/). 